\title{
Detection of Hemorrhage from Fundus Images using Hybrid Method
}

\author{
Reshma M. Mulla \\ PG Student, Department of Electronics \\ Engineering \\ K.I.T's college of Engineering, Kolhapur, India
}

\author{
Chavan M.S. \\ Professor, Department of Electronics Engineering \\ K.I.T's college of Engineering, Kolhapur, India
}

\begin{abstract}
Diabetic retinopathy is serious health issue in recent survey. Diabetic disease occurs when insulin level in blood vessels decreases and body is unable to process properly. if diabetic increases then it effect the retina of fundus.and this disease damage the blood vessels and damage the retina. The aim of this study to detect blood vessels, identify hemorrhage and classify diabetic retinopathy in normal,moderate and proliferative stage. There are two levels of diabetic retinopathy non proliferative and proliferative stage.non proliferative is first stage of diabetic retinopathy which include micro aneurysms and cotton wool spots. if it is increases then it enters into proliferative stage. In past days hemorrhage detected manually pixel by pixel method. In this paper developed automated method for detection of hemorrhage. in which used template matching technique for the detection of hemorrhage. For detection of proper size of hemorrhage region growing segmentation used in this method. for elimination of false positive fovea filter used this will eliminate wrongly detected blood vessels as hemorrhage. This study is improving our automated hemorrhage detection method to help diagnosis of diabetic retinopathy. The sensitivity for the detection of abnormal cases were $80 \%$ and $90 \%$ respectively. These results indicate that this new method may improve the performance of diagnosis system for detection of hemorrhage.
\end{abstract}

\section{General Terms}

Template matching, region growing segmentation et. al.

\section{Keywords}

Diabetic, hemorrhage,fundus image, detection ,template

\section{INTRODUCTION}

Diabetic occurs due to low level of insulin in blood vessels and this will effect the retina of eye.recent survey shows that many people suffer from diabetes timely and proper treatment can prevent eyes from damage.for diagnosis of diabetic fundus images capture by fundus camera for the detection of hemorrhage this fundus images of retina are used.our aim of this study to identify blood vessels correctly, detection of hemorrhage and classify the stages of diabetic retinopathy. The preliminary sign of diabetic retinopathy micro aneurysm,hemorrhage. There are two levels of diabetic retinopathy non-proliferative and proliferative. nonproliferative is first stage of diabetic retinopathy. Micro aneurysm occurs in this stage. The blood vessels becomes blocked and leads into retinal hemorrhage and comes into proliferative stage. so timely and proper treatment can prevent eyes from damage. In past days hemorrhage counted pixel by pixel method. it is very time consuming and lengthy process. and accuracy of this manually detection is less. so automated detection method detect the hemorrhage in diabetic retinopathy and it improve the performance of computer-aided diagnosis of hemorrhage in DR.so the computer aided system can help accurate detection of DR which can prevent eyes from blindness. this automated detection of hemorrhage fundus images used which are captured by fundus camera.

\section{CASE STUDY}

\subsection{Background of Work}

For early diagnosis of diabetic retinopathy detection of hemorrhage is most important factor.recent survey indicate that many people suffer from diabetic and this will effect on the retina. This disease can be prevented by giving examination and treated in early stages.fundus photographs obtained by fundus camera used for diagnosis of DR. Jang pyo Bae et.al has presented that extraction of hemorrhages using template matching with templates of various shapes. he applied hue saturation value brightness correction and contrast-limited adaptive histogram equalization to fundus images.[1]Giri babu kande et.al has conferred an economic formulation for autoloading sensing of red lesions in fundus image based on pixel classification \& numerical morphology is proposed. [2]Yuji Hatanka et.al has given a bran-new method for detecting hemorrhage which may effectively change the performance of our computer-aided diagnosing system for hemorrhages. It has built automatic hemorrhage detection method to help analyze diabetic retinopathy. $\mathrm{He}$ design a new method in which a brightness of the fundus image was varied by the nonlinear curve with brightness values of the hue saturation value (HSV) space. [3]Alan D.fleming et.al has conferred that automatic methods for MA detection and appearance how image contrast normalization can modify the quality to separate between MAs and other dots that occur on the retina. Effect are presented for detection of respective MAs and for detection of images containing MA.[4]R.Vidyasari et.al has given that there are two stages of diabetic retinopathy which are nonproliferative diabetic retinopathy (NPDR) and proliferative diabetic retinopathy (PDR).The presence of micro aneurysms in the eye is one of the primal signs of diabetic retinopathy. [5]

\subsection{Problem Definition}

Diabetic retinopathy is very serious disease in medical system.if level of insulin is less in blood vessels then diabetic occurs. Because of this blood vessels of retina are broken and bleed comes white part of retina and this leakage of bleed is called hemorrhage, It is progressive eye disease, if taking proper treatment on this disease can prevent eyes from blindness. so detection of hemorrhage in early stage is most important to prevent diabetic patient from blindness

\subsection{Objectives of Case Study}

To accomplish detection of diabetes retinopathy there are various methods used. After classifying the well known methods of detection hemorrhage candidate will be extracted. The beginning signs of diabetic retinopathy include micro 
aneurysms, hemorrhages. early treatment and proper diagnosis can prevent eyes from blindness. Considering the vast scope for the research in this area the following objective are defined for the proposed work.

1) Theoretical study of method for detection of hemorrhage and diabetes retinopathy.

2) Collection of fundus Images (Developing data base)

3) To Study \& analysis of the method.

I. Hybrid method.

4) Performance Evaluation of method.

5)To study classification of different stages of diabetic retinopathy.

7)Based on detection of hemorrhage classify different stages of diabetic retinopathy as normal, moderate and nonproliferative diabetic retinopathy (NPDR)

\section{PROPOSED METHODOLOGY}

1. Study of method for detection of hemorrhage \& diabetic retinopathy.

2. Collection of more than 100 fundus Images as database.

3. Study and analysis of hybrid method for detection of hemorrhage in diabetic retinopathy.

4. Apply method on fundus images.

5. Performance evaluation of method.

6. To study the classification of different stages in diabetic retinopathy.

7.Based on detection of hemorrhage classify different stages of diabetic retinopathy as normal, moderate and nonproliferative diabetic retinopathy (NPDR).

\section{HYBRID METHOD}

\subsection{Introduction}

For the detection of hemorrhage in diabetic retinopathy image processing used..In this preprocessing process used for enhancing the contrast between background and object. For extraction of hemorrhage in fundus image after preprocessing template maching technique used.in which template of various shapes are used for detection of hemorrhage.for extract the proper shape of hemorrhage region growing segmentation technique used.

\subsection{Flowchart of Detecting Hemorrhage}

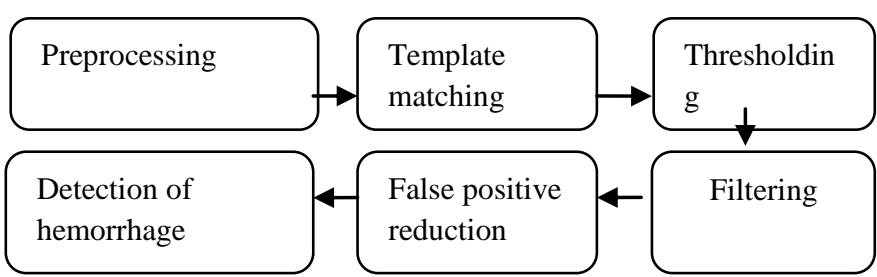

Figure 1: Block diagram of Hybrid method

\subsection{Preprocessing}

A digital image is composed of red, green and blue channel In this hybrid method green channel used because it represent red structure well and it is less noisy then red and blue channel .preprocessing mainly used for two processes first for making the brightness of image and second is enhancing the contrast between background and hemorrhage. For enhance the contrast of image program can use CLAHE algorithm and general contrast algorithm.[1]
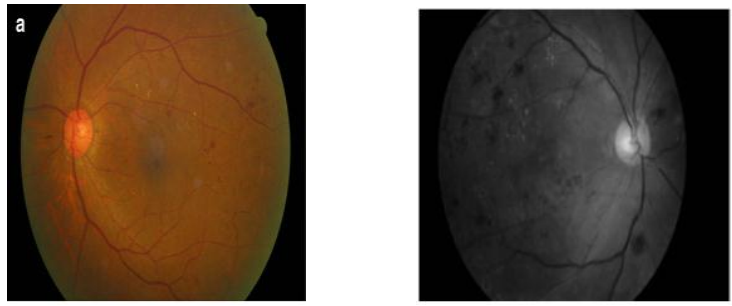

Figure2:(a)Original fundus image (b) Green plane image[1]

\subsection{Contrast-Limited Adaptive Histogram Equalization}

To improve the contrast of image adaptive histogram equalization technique used in image processing. each related to a different segment of the image, and uses them to sort out the lightness values of the image. Therefore it is proper for improving the local contrast of an image and bringing out more detail. A variant of adaptive histogram equalization called contrast limited adaptive histogram equalization (CLAHE) prevents this by restricting the amplification. The form of a picture is rectangular. However, the shape of an eye is round because the rectangular representation contains the round eye; in the fundus image, the dark outside surface part which surroundings an eye appears. In general-purpose histogram equalization, the picture element of dark outside region is added to the histogram, so values of element close this dark outside region were less than the expected ones. CLAHE work on tiny regions, called tiles, while the generic algorithmic program works on the whole image. As the result of extremely dark and bright regions is limited to the local tile, a uniform image can be occur.[1]

\subsection{Template Matching}

Detection of template matching technique used after applies the CLAHE algorithm. for the detection multiple size of template used because single template cannot cover the proper hemorrhage. Fig. 3 shows that shape of template in which radius denote by (r) and outside width denote by(a).If radius of circle greater then large number of hemorrhage detected and if outside width is greater than more background included. So gathered hemorrhage are missed out for this shorter outside width used. Independent hemorrhage can be easily detected.
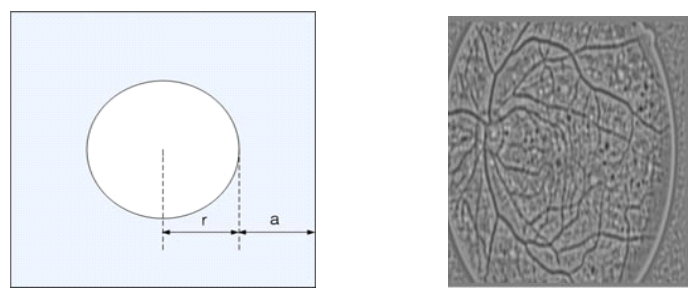

Figure 3: (a)Tamplate Matching (b) Result of template matching[1]

\subsection{Region Growing Segmentation}

After using template matching we get only information about center point of an object .so proper size and lacks of information of object recovered by using region growing segmentation .regions of image segmentation used for find regions of objects and important parts of objects. The main goal of segmentation is to partitioning an image into parts. Some segmentation methods such as thresholding accomplish 
this goal by looking for the edge between regions based on discontinuities in gray scale or color properties.

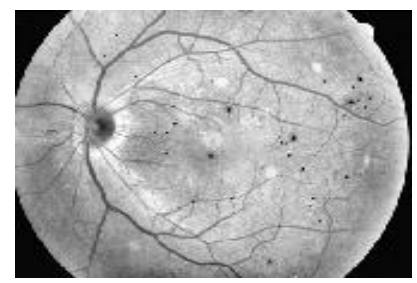

Figure 4: Image of region growing segmentation[1]

\subsection{Region Growing Segmentation using the Local Threshold}

Thresholding is the simplest segmentation technique. With a single threshold, it change a gray scale or color image into a binary image.. From a gray scale image, thresholding can be used to create binary images.

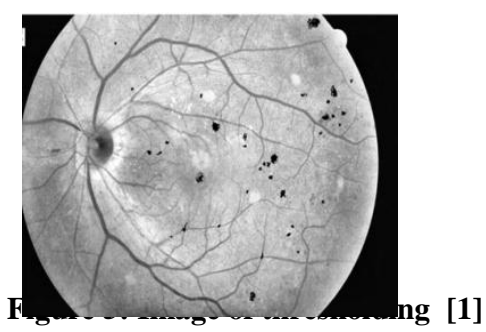

\subsection{False Positive Reduction}

After region growing there is some false positive element present in image. Wrongly detected hemorrhage is known as false positive. For elimination of FP area feature twisely used. This eliminates tiny spots chosen from template matching, and candidates whose sizes are lower than the area threshold were expelled. After region growing recovers the accurate form of hemorrhage, for elimination of false positive area of hemorrhage used. The formation of the fundus image can provide us large information about the hemorrhage detection. six filters are used to classify each one of candidate hemorrhage as either a red lesion or a non-red lesion,. In first set of filters size ,shape and compactness of hemorrhage in fundus images are used $.5 \times 5 \mathrm{kernel}$ value and fovea filter are used as second set of filters.

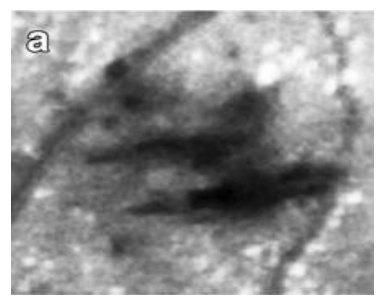

Figure 6: (a) wrongly detected hemorrhage [1]

\section{PERFORMANCEANALYSIS PARAMETER}

For the performance evaluation used five parameter True positive (TP), True negative(TN),False positive(FP),False Negative(FN),Sensitivity.

1) True positive - The system detects the symptoms correctly is called True positives.

2) True negative - The system cannot detect the symptoms correctly is called True negative.
3) False positive - The systems detect the symptoms but it is not the symptoms is called False positive.

4) False negative - The system cannot detects the presence of symptoms is called False negative.

5) Sensitivity -The sensitivity measures the proportion of actual positives which are correctly identified.

$\mathrm{TP} /(\mathrm{TP}+\mathrm{FN})$

\section{RESULTS FOR HYBRID METHOD 6.1 Hybrid method}

Table 1.Performance of preprocessing

\begin{tabular}{|l|l|l|l|l|l|}
\hline & Tp & Tn & Fp & Fn & Sensitivity \\
\hline no preprocessing & & & & \\
\hline image 1 & 3 & 0 & 2 & 0 & 1.0 \\
\hline image 2 & 8 & 4 & 1 & 0 & 1.0 \\
\hline Clahe & 3 & 2 & 3 & 0 & 1.0 \\
\hline image 1 & 1 & 4 & 1 & 4 & 0.2 \\
\hline image 2 &
\end{tabular}

Table 2 Results of fovea filter

\begin{tabular}{|l|l|l|l|l|l|}
\hline & Tp & Tn & Fp & Fn & sensitivity \\
\hline Before & & & \\
\hline image 1 & 2 & 11 & 20 & 0 & 1 \\
\hline image 2 & 0 & 1 & 21 & 0 & 0.8 \\
\hline After & 2 & 0 & 0 & 0 & 1 \\
\hline image 1 & 2 & 0 & 0 & 0 & 0.9 \\
\hline image 2 & 0 & 0 &
\end{tabular}

Table 3.Results of varing threshold value

\begin{tabular}{|c|c|c|c|c|c|}
\hline Threshold & Tp & Tn & Fp & Fn & Sensitivity \\
\hline 4 & 9 & 1 & 1 & 2 & 0.8 \\
\hline 7 & 40 & 3 & 3 & 4 & 0.7 \\
\hline 10 & 10 & 0 & 0 & 4 & 0.7 \\
\hline 15 & 16 & 2 & 3 & 4 & 0.6 \\
\hline 20 & 2 & 6 & 6 & 1 & 0.6 \\
\hline
\end{tabular}

\subsection{Preprocessing performance}

The general contrast enhancement algorithm was compared to the CLAHE algorithm combined with HSV brightness correction. The general contrast enhancement algorithm generated an irregular image Due to the optic disk and the dark outside area. CLAHE solved this problem and the number of FPs per image of CLAHE algorithm was lower than that of the other algorithms in Table 1.

\subsection{Filter performance}

Results of the foveal filter were shown in Table 2. "Before" is the test result before application of the foveal filter. "Foveal filter result" is the test result after application of the foveal filter. To recover the big missed hemorrhages from the FNs; a kernel compensation technology was used. before used filter there was large number of missed hemorrhage were detected. 
But after using fovea filter FP per image is decreased. so the sensitivity also better than before.

\subsection{Thresholding performance}

Results of thresholding shown in table 3.there was varied area threshold as 4, 7, 10, 15, and 20, 25 in this if threshold value was increased then sensitivity decreased. If threshold value was high then FP per image was less. And also sensitivity better than increased value of threshold. If FP of image was less then sensitivity was high. And if FP was high then sensitivity was decreased. The curve show that FP verses sensitivity graph. It was a show that if FP was low then sensitivity was high. and if FP was high then sensitivity was low.

\section{CLASSIFICATION \\ 7.1 Hybrid method}

Classification of DR is based on number of MA and hemorrhage present in the fundus images.In table shows that if there was no any abnormality then there was no apparent retinopathy.if there was microaneurysms present in fundus image then this was classified as mild NPDR. If More than just MA but less than severe NPDR,less then 20 hemorrhage then classified as moderate NPDR. Definite bleeding, sign of proliferative, Greater then 20 hemorrhage then it was classified as severe NPDR.if there was retinal hemorrhage and definite bleeding then it was classified as severe PDR.

Table 4: Classification of fundus images

\begin{tabular}{|l|l|l|}
\hline Images & $\begin{array}{l}\text { No of } \\
\text { hemorrhage }\end{array}$ & Classification \\
\hline Image 1 & 10 & Moderate NPDR \\
\hline Image 2 & 22 & Severe NPDR \\
\hline Image 3 & 5 & Moderate NPDR \\
\hline Image 4 & 25 & Severe \\
\hline
\end{tabular}

\section{CONCLUSION}

Diabetic retinopathy is a severe difficulty of diabetes mellitus and a important reason of loss of sight in widereaching. The identification of hemorrhages is one of the essential element in the early on analysis of diabetic retinopathy (DR).recognition of hemorrhages in diabetic retinopathy it is main diagnostic standard to find out the severity of diabetic retinopathy. Detection of hemorrhage it is extremely important to prevent our eyes in diabetic retinopathy.a new method for automatically detecting hemorrhages it was implanted that the algorithmic program detected abnormalities with higher accuracy and reliability. a new scheme for automatically detecting hemorrhages and their exudates is presented by using digitized ocular fundus images as an example. This scheme can be applied to the computer-aided diagnosis (CAD) system for diagnosing diseases detected in the eyes. The results of the preliminary testing showed a desirable consistency with those obtained from the proposed scheme. It was demonstrated that the algorithm detected abnormalities with higher accuracy and reliability. The result of the initial work on fundus images clarified that the efficiency and accuracy of diabetic retinopathy diagnosis was considerably improved. The results of this study will be sent to ophthalmologists for further evaluation. The efficiency and accuracy diagnosing of diabetic retinopathy was improved due to the detection of hemorrhages and exudates with a higher accuracy. The application of the proposed scheme to fundus images enhances the CAD system performance for detecting hemorrhages and exudates in the fundus images. For the future work, we need to detect other symptomps such as cotton wool spots, exudates, and veanous beading because they also important factor in the classification of NPDR. Also, we need to check the location of the microaneurysms, especially microaneurysms located near thevessels.

\section{REFERENCES}

[1] Jang Pyo Bae, Kwang Gi Kim, Ho Chul Kang, Chang Bu Jeong,Kyu Hyung Park" A Study on Hemorrhage Detection Using Hybrid Method in Fundus Images" Hwang Journal of Digital Imaging, Vol 24, No 3 (June), 2011

[2] Giri Babu Kande, T. Satya Savithri, and P. Venkata Subbaia ,"Automatic Detection of Microaneurysms and Hemorrhages in Digital Fundus Images," Journal Of Digital Imaging, Vol 23, No 4 (August), 2010

[3] Hatanaka Y, Nakagawa T, Hayashi Y, Hara T, Fujita H:'Improvement of Automated Detection Method of Hemorrhages in Fundus Images." IEEE EMBS Vancouver, Canada, 2008

[4] Alan d, Fleming, Sam Philip, Keith A. Goat man, John A. Olson, and Peter F. Sharp. ," Automated Micro aneurysm Detection Using Local Contrast Normalization and Local Vessel Detection" IEEE transactions on medical imaging, vol. 25, no. 9, september 2006

[5] R. Vidyasari , I. Sovani, and T.L.R. Mengko ,H. Zakaria," Vessel Enhancement Algorithm in Digital Retinal Fundus Micro aneurysms Filter for Nonproliferative Diabetic Retinopathy Classification"November 2011, Bandung, Indonesia.

[6] Sunrita Poddar, Bibhash Kumar Jha, Chandan Chakraborty," Quantitative Clinical Marker Extraction from Color Fundus Images for Non-Proliferative Diabetic Retinopathy Grading” 2011 India.

[7] Toshiaki Nakagawa, Yoshinori Hayashi," Improvement of Automated Detection Method of Hemorrhages in Fundus Images.'Dept.of Electronic Control Engineering,Vol. 6915, 69153E, (2008)

[8] Mulla R.M,Chavan M.S.,"Performance evaluation of Differant methods for Detection of Hemorrhage Using Fundus images,",International Journal of Inovative research in Science,Engineering and Technology vol.3,Issue 9,September 2014,

[9] Kanika Verma, Prakash Deep and A. G. Ramakrishna," Detection and Classification of Diabetic Retinopathy using Retinal Images "Bangalore, India.

[10] Divyanjali Satyarthi, B.A.N. Raju, and S. Dandapat." Detection of Diabetic Retinopathy in Fundus Images using Vector Quantization Technique"Indian Institute of Technology Guwahati, Guwahati-781039, Assam, India.

[11] Meindert Niemeijer, Michael D. Abramoff, Member, IEEE, and Bram van Ginneken, Member," Information Fusion for Diabetic Retinopathy CAD in Digital Color Fundus Photographs"IEEE transactions on medical imaging, vol. 28, and no. 5, may2009

[12] X. Zhang and G. Fan, "Retinal Spot Lesion Detection Using Adaptive Multiscale Morphological Processing", in Proc. ISVC (2), 2006. 
[13] Parisut Jitpakdee, Pakinee Aimmanee, Bunyarit Uyyanonvara,"A Survey on Hemorrhage Detection in Diabetic Retinopathy Retinal Images" Sirindhom International Institute of Technology Thammasat University, 2012 IEEE Bangkok,

[14] Matei, Daniela, and Radu Matei Langroudi, M.N., Sadjedi, H. "Detection of diabetic symptoms in retina images using analog algorithms." International Journal of Biological and Life Sciences 6.4 (2010)
[15] Acharya UR, Lim CM, Ng EY, Chee C, Tamura T., "Computer-based detection of diabetes retinopathy stages using digital fundus images",2009.

[16] HT Nguyenl, M Butler A Roychoudhryl, AG Shannonl, J Flack, P Mitchell," Classification of diabetic retinopathy using neural networks" Australia. 